\title{
An EMG-Controlled Omnidirectional Pointing Device Using a HMM-based Neural Network
}

\author{
Osamu Fukuda ${ }^{1}$, Jun Arita $^{2}$ and Toshio Tsuji ${ }^{2}$ \\ ${ }^{1}$ National Institute of Advanced Industrial Science and Technology, \\ Tsukuba,Japan, fukuda.o@aist.go.jp \\ ${ }^{2}$ Hiroshima University, Higashi-hiroshima, Japan
}

\begin{abstract}
This paper proposes a new EMG-controlled pointing device using a novel statistical neural network. This device can be used as an interface tool for wearable computers since it does not restrict the operator to being in front of computer devices such as a keyboard or a mouse.

The distinctive feature of this device is that we adopt a statistical neural network, which includes a continuous density hidden Markov model, to model the relationship between EMG signals and directions of a pointer movement. The operator can move a pointer in any direction throughout 360 degrees. We also introduced a physical model, such as a mass in a viscous space, into our system to realize a smooth pointer movement corresponding to the operator's force sense. In the experiments, omnidirectional pointer control is achieved using the proposed method and the applicability of our method is confirmed.
\end{abstract}

\section{INTRODUCTION}

The quantity of information dealt with by human operators is rapidly increasing with the development of information technology. A new interaction channel is required to realize advanced man-machine communications with the development of various new devices such as wearable computers, and mobile devices. As new interface channels, we should adopt not only external expressions such as voices and gestures, but also internal physiological information from human bodies. Also, we should consider that the disabled need new interface devices. Since it is very difficult for the disabled, especially amputees, to use current interface devices, the development of new interface devices is expected. If the amputee can use personal computers and Internet technologies using the new interface devices, these devices will play important roles in providing various information and improving their quality of life (QOL).
Bioelectric signals such as EEG signals and EMG signals can be expected to be used as new interface channels. These signals include various important information that cannot be observed from outside human bodies. For example, EEG signals include information concerning intention, awaking and mental stress, and EMG signals include information concerning muscle force, intended motion and muscle impedance. If the hidden information are estimated accurately, these signals will be useful as new interaction channels. They are especially important for seriously handicapped people as they may represent their only hope in restoring the intended functions.

Up to the present, several methods for the EEG and EMG pattern discrimination using neural networks have been proposed [1]-[6]. In the first stage of this study, Kelly et al. [1] proposed a pattern discrimination method combining the back propagation neural network (BPN) and the Hopfield neural network. However, BPN cannot realize high learning and discrimination performance because the EMG patterns differ considerably at the start and end of the motion even if they are within the same class. While the BPN was utilized in most of the previous studies, the authors [3], [4] proposed a statistical neural network called Log-Linearized Gaussian Mixture Network (LLGMN) based on a log-linear model and a Gaussian mixture model, and realized higher discrimination performance than that of other neural networks. Also, an EMG-controlled pointing device was proposed using LLGMN, and the performance of this method was reported [5], [6]. This method can control the pointer in an arbitrary direction, but accuracy of the estimated direction was not so high. Tsuji et al. [7] has therefore proposed Recurrent LogLinearized Gaussian Mixture Network (R-LLGMN) based on a continuous density hidden Markov model (CDHMM) [8]. This network uses recurrent connections added to the units of LLGMN in order to discriminate a time sequence of the signals with high accuracy. 
This paper proposes a new EMG-controlled omnidirectional pointing device using R-LLGMN. In the proposed pointing device, an arbitrary direction of pointer movement is represented using a combination of finite base directions. The probability of pointer movements in each base direction can be estimated by R-LLGMN using probability theory.

\section{RELATED WORKS}

Research on a human-computer interface using EMG signals and EEG signals has been conducted. For example, Rosenberg [9] used EMG signals as an interface for wearable computers and investigated pointing in a two-dimensional display. This research, however, used the conventional BPN and enables only $14 \%$ of performance compared with performance of a conventional computer mouse based on Fitts's law [10]. Barreto et al. [11] developed a computer interface using EMG signals and EEG signals in order to support the disabled. In this research, they realized four-directional pointing and click operations using EMG signals measured from facial muscles and using the frequency information of the EEG signals. Wolpaw et al. [12], Roberts et al. [13], and Mason et al. [14] reported pointing operation using only the EEG signals. Robert et al. discriminated two commands from 1ch EEG signals. Mason et al. controlled switches depending on EEG features. However, the systems in these studies were unable to adapt to the operator's characteristics caused by the disabled part, fatigue, aging, and so on, so that it is very difficult to employ them in practical applications.

Control performance is the most important problem for developing interface devices. In most previous research, the multiple degree of freedom (DOF) control problem corresponds to the discrimination problem of EMG patterns, so that the DOF is limited to the class number in pattern recognition. In such a case, we must define the number of the possible directions (e.g, up, down, right, left) beforehand to discriminate pointer movement directions. In addition, the increasing number of defined movements will require the learning and discrimination of an enormous number of patterns. If we use the BPN to learn these patterns, a very large number of learning iterations will be required or local minima problems may arise. We should design a new strategy for EMG pattern discrimination to develop new omnidirectional pointing devices.

\section{SYSTEM COMPONENTS}

Realizing the proposed pointer control requires the following techniques.
1. Since the EMG signals include high-frequency components, we must perform adequate signal processing such as rectifying, filtering, and normalizing to extract meaningful information for the human interface.

2. Adaptation learning ability and high discrimination performance are needed to adapt the system to the operator's characteristics such as control ability and accuracy and to estimate the operator's intended direction of the pointer movement with high accuracy.

3. To realize smooth movement of a pointer corresponding to the operator's force sense, the system should incorporate a control method based on a physical model, such as a mass in a viscous space.

The structure of the proposed system, which incorporates the three points above, is shown in Fig. 1. This system consists of the EMG signal processor, the neural network, and the pointer controller. Details of each part are explained in the following subsections.

\subsection{EMG Signal Processor}

The EMG signal processor extracts features for pointer control from the EMG signals. First, the EMG signals which are measured from $(L+1)$ pairs of electrodes (Web5000: NIHON KOHDEN Corp.) are amplified and digitized by an A/D converter. Then, they are rectified and filtered out through the second order Butterworth filter (cut-off frequency: 1[Hz]), and re-sampled with a $100[\mathrm{~Hz}]$ sampling frequency. $L$ sets of the $E M G_{i}(t)$ measured from the channels, except for the $(L+1)$-th channel, are utilized to represent the direction and velocity of the pointer movement. In order to estimate the direction of pointer movement, $E M G_{i}(t)$ are normalized to make the sum of $L$ channels equal to 1 by the following definition:

$$
x_{i}(t)=\frac{E M G_{i}(t)-\overline{E M G}_{i}^{s t}}{\sum_{i^{\prime}=1}^{L}\left(E M G_{i^{\prime}}(t)-\overline{E M G}_{i^{\prime}}^{s t}\right)},
$$

where ${\overline{E M G_{i}}}^{\text {st }}$ is the mean value of $E M G_{i}(t)$ while relaxing the muscle. This is necessary to extend the EMG pattern independent of the amplitude of the EMG signals that are highly depending on the force level. $x_{i}(t)$ is an element of the feature vector $\boldsymbol{x}(t)=$ $\left[x_{1}(t), x_{2}(t), \cdots, x_{L}(t)\right]^{\mathrm{T}} \in \Re^{L}$. This vector indicates the cooperation pattern among several muscles and 


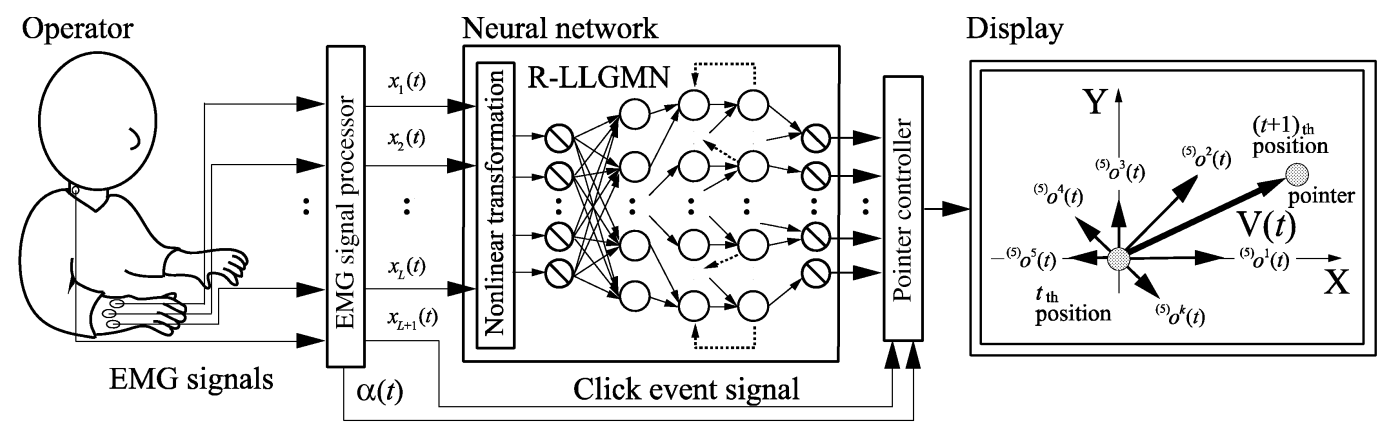

Figure 1: Structure of the proposed system.

is used as the input pattern of the neural network to estimate the direction of the pointer movement.

The signal from the $(L+1)$-th channel is employed to process the click event in the operation. The EMG signal from the $(L+1)$-th channel, $E M G_{L+1}(t)$, is measured in a different part of a body (e.g, the neck) from signals of other channels. When the value of $E M G_{L+1}(t)$ exceeds the prespecified threshold, it is determined that a click event has occurred.

\subsection{Neural Network [7]}

In the neural network, the direction of the pointer movement is estimated from the EMG pattern $\boldsymbol{x}(t)$ extracted by the EMG signal processor. To achieve reliable GUI operation, it is necessary to control the direction of pointer movements accurately according to the intention of the operator. However, if the pointer is allowed to move in all directions from the current position, the number of moving directions will be infinite. Consequently, in the proposed system, a finite number of base directions is shown on the computer display, and the operator's intended direction is estimated from the probability that the pointer will move to each base direction. The moving direction of the pointer is calculated from the estimated results for all base directions.

For example, in Fig. 1, the moving direction is estimated as the bold arrow by using $K$ base directions $(1,2, \cdots, K)$. An arbitrary intended direction can be represented with a set of arrows corresponding to the probability of the pointer movement to a finite number of base directions. Since the neural network utilized in this system only estimates the probability for each base direction, it may lead to avoid a heavy learning calculation and a huge network structure.

R-LLGMN [7] is used for the estimation. R-LLGMN can acquire CDHMM [8] through learning and can calculate the a posteriori probability of the pointer movement according to this model. The probability density function is expressed by the weighted sum of the Gaussian components. It enables the R-LLGMN to learn the complicated mapping between the operator's EMG patterns and the pointer movements.

The structure of R-LLGMN is shown in Fig. 2 . The five-layered structure with feedback connections between the fourth layer and the third layer is of good correspondence with the algorithm of CDHMM. There are $C$ classes and each class $c(c \in$ $1, \cdots, C)$ is composed of $K_{c}$ states. The a posteriori probability, which is calculated in the class $c$ and the state $k$, is approximated by summing up $M_{c, k}$ components of a Gaussian mixture distribution. However, R-LLGMN is not just a copy of CDHMM. The essential point of R-LLGMN is that the parameters in CDHMM are replaced by the weight coefficients $w_{k^{\prime}, k, m, h}^{c}$, and this replacement removes restrictions of the statistical parameter in CDHMM (e.g., $0 \leq$ the transition probability $\leq 1$, and standard deviations $>0$ ). Therefore, the learning algorithm of R-LLGMN is simplified and can be expected higher generalization ability than that of CDHMM. That is the great advantage of R-LLGMN.

First of all, the input vector $\mathbf{x}(t)=\left[x_{1}(t), x_{2}(t), \cdots\right.$, $\left.x_{d}(t)\right]^{\mathrm{T}} \in \Re^{d}(t=1, \ldots, T)$ is pre-processed with a non-linear computation and converted into the modified vector $\mathbf{X} \in \Re^{H}$ :

$$
\begin{aligned}
\mathbf{X}(t)= & {\left[1, \mathbf{x}(t)^{\mathrm{T}}, x_{1}(t)^{2}, x_{1}(t) x_{2}(t), \cdots,\right.} \\
& x_{1}(t) x_{d}(t), x_{2}(t)^{2}, x_{2}(t) x_{3}(t), \\
& \left.\cdots, x_{2}(t) x_{d}(t), \cdots, x_{d}(t)^{2}\right]^{\mathrm{T}} .
\end{aligned}
$$

This nonlinear transformation is needed to represent the probability density function corresponding to each component of the GMM in CDHMM's state as a linear combination of the new input vector $\boldsymbol{X}(t)$. The first layer consists of $H=1+d(d+3) / 2$ units and the identity function is used for activation of each unit. Unit $\left\{c, k, k^{\prime}, m\right\} \quad(c=1, \cdots, C$; $\left.k, k^{\prime}=1, \ldots, K_{c} ; m=1, \ldots, M_{c, k}\right)$ in the second 


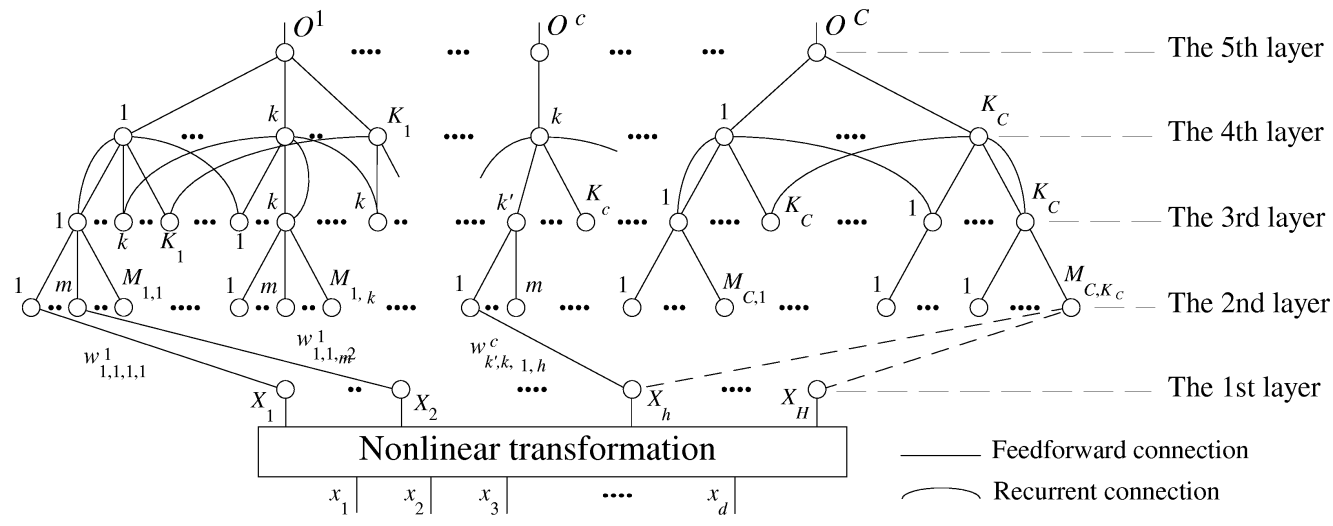

Figure 2: The structure of R-LLGMN

layer receives the output of the first layer ${ }^{(1)} O_{h}(t)$ corresponding to $X_{h}(t)(h=1,2, \cdots, H)$ weighted by the coefficient $w_{k^{\prime}, k, m, h}^{c}$. The relationship between the input and the output in the second layer is defined as

$$
\begin{aligned}
& { }^{(2)} I_{k^{\prime}, k, m}^{c}(t)=\sum_{h=1}^{H}{ }^{(1)} O_{h}(t) w_{k^{\prime}, k, m, h}^{c}, \\
& { }^{(2)} O_{k^{\prime}, k, m}^{c}(t)=\exp \left({ }^{(2)} I_{k^{\prime}, k, m}^{c}(t)\right),
\end{aligned}
$$

where $C$ is the number of classes corresponding to the number of the base directions, $K_{c}$ is the number of states, $M_{c, k}$ is the number of the components of the Gaussian mixture distribution in the class $c$ and the state $k$.

The input into a unit $\left\{c, k, k^{\prime}\right\}$ in the third layer integrates the outputs of units $\left\{c, k, k^{\prime}, m\right\} \quad(m=$ $\left.1, \ldots, M_{c, k}\right)$ in the second layer. The output in the third layer is that input weighted by the previous output in the fourth layer. The relationship in the third layer is defined as

$$
\begin{gathered}
{ }^{(3)} I_{k^{\prime}, k}^{c}(t)=\sum_{m=1}^{M_{c, k}}{ }^{(2)} O_{k^{\prime}, k, m}^{c}(t), \\
{ }^{(3)} O_{k^{\prime}, k}^{c}(t)={ }^{(4)} O_{k^{\prime}}^{c}(t-1)^{(3)} I_{k^{\prime}, k}^{c}(t),
\end{gathered}
$$

where ${ }^{(4)} O_{k^{\prime}}^{c}(0)=1.0$ for the initial state.

The fourth layer receives the integrated outputs of units $\left\{c, k, k^{\prime}\right\}$ in the third layer. The relationship in the fourth layer is defined as

$$
{ }^{(4)} I_{k}^{c}(t)=\sum_{k^{\prime}=1}^{K_{c}}{ }^{(3)} O_{k^{\prime}, k}^{c}(t)
$$

$$
{ }^{(4)} O_{k}^{c}(t)=\frac{{ }^{(4)} I_{k}^{c}(t)}{\sum_{c^{\prime}=1}^{C} \sum_{k^{\prime}=1}^{K_{c^{\prime}}}(4) I_{k^{\prime}}^{c^{\prime}}(t)} \text {. }
$$

At last, a unit $c$ in the fifth layer integrates the outputs of $K_{c}$ units $\{c, k\}\left(k=1, \ldots, K_{c}\right)$ in the fourth layer. The relationship in the fifth layer is defined as

$$
\begin{gathered}
{ }^{(5)} I^{c}(t)=\sum_{k=1}^{K_{c}}{ }^{(4)} O_{k}^{c}(t), \\
{ }^{(5)} O^{c}(t)={ }^{(5)} I^{c}(t) .
\end{gathered}
$$

The output of the network ${ }^{(5)} O^{c}(t)$ indicates the $a$ posteriori probability of pointer movement to the base direction $k$ (see Fig. 1).

R-LLGMN must be learned before the operation. In the learning process, the specified direction of the pointer movement $\phi(0 \leq \phi<2 \pi)$ is indicated to an operator on the display, and the operator is asked to generate the EMG signals according to this direction. The order of presenting the specified direction $\phi$ is random, where $\phi$ is defined as 0 in the positive direction of the $X$-axis and increases counterclockwise. The $N$ pairs of the EMG patterns and the specified direction $\phi$ are then used as the learning data.

\subsection{Pointer Controller}

The pointer controller determines the direction and the speed of the pointer movement. The direction of the pointer movement is estimated using the output of the neural network. The output of the RLLGMN ${ }^{(5)} O^{c}(t)$ indicates the a posteriori probability that the pointer will move to each base direction $k$ as shown in Fig. 1. Therefore, the direction of the pointer movement by the $t$-th EMG pattern, $\boldsymbol{e}(t)=\left(e_{X}(t), e_{Y}(t)\right)^{\mathrm{T}}$, is defined as follows: 


$$
\begin{gathered}
e_{X}(t)=\frac{v_{X}(t)}{\sqrt{v_{X}^{2}(t)+v_{Y}^{2}(t)}}, \\
e_{Y}(t)=\frac{v_{Y}(t)}{\sqrt{v_{X}^{2}(t)+v_{Y}^{2}(t)}}, \\
v_{X}(t)=\sum_{k=1}^{K}{ }^{(5)} O^{c}(t) \cos (2 \pi(k-1) / K), \\
v_{Y}(t)=\sum_{k=1}^{K}{ }^{(5)} O^{c}(t) \sin (2 \pi(k-1) / K) .
\end{gathered}
$$

To control the speed of the pointer, the muscular contraction level $\alpha(t)$ is calculated as

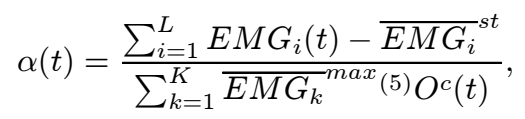

where ${\overline{E M G_{i}}}^{\text {st }}$ is the mean value of $E M G_{i}(t)$ while relaxing the muscle; and $\overline{E M G}_{k}^{\max }$ is the mean value of $\sum_{i=1}^{L} E M G_{k i}^{\max }(t)$, where $E M G_{k i}^{\max }(t)(k=$ $1, \cdots, K)$ is measured for base direction $k$ while keeping the maximum voluntary construction (MVC) beforehand. This muscular contraction level is utilized to regulate the difference among the levels for each direction in MVC.

In order to realize smooth pointer movement corresponding to the operator's intended direction and force sense, we use the following physical model based on $\alpha(t)$ and $\boldsymbol{e}(t)$ :

$$
M_{e} \ddot{\boldsymbol{p}}(t)+B_{e} \dot{\boldsymbol{p}}(t)=\boldsymbol{F}(t),
$$

where $M_{e}$ and $B_{e}$ denote the inertia and the viscosity parameter, respectively; and $p(t)$ is the position vector of the pointer. $\boldsymbol{F}(t)$ is the drive force to the pointer depending on $\alpha(t)$ and $\boldsymbol{e}(t)$.

$$
\boldsymbol{F}(t)=\left\{\begin{array}{ll}
g \alpha(t) \boldsymbol{e}(t) & \left(\alpha(t) \geq \alpha_{0}\right) \\
0 & \left(\alpha(t)<\alpha_{0}\right)
\end{array},\right.
$$

where $g$ and $\alpha_{0}$ are the force gain and the threshold that determine whether the pointer has been operated or not. Equation (16) expresses the dynamic motion equation of an object (weight $M_{e}$ ) in viscous space with force $\boldsymbol{F}(t)$ and viscous friction $B_{e}$. The position and velocity of the pointer are calculated by numerical integration depending on such the physical model, so that it can be expected to realize a natural feeling of control corresponding to the force sense of an operator.

\section{EXPERIMENTS}

To examine the feasibility of the proposed method, we conducted pointer control experiments. Seven electrodes ( $L=6$ : six in the right forearm and one in the left forearm) were used, and the direction of the pointer was aligned to the bending direction of the right wrist of the subject. The information on click events was extracted from the EMG signal measured at the left forearm. The length of the training data was $T=20$, and 2 samples of the learning data were used for each base direction.

Figure 3 (a)(b) shows an example of the pointer control. To confirm omnidirectional control ability, the operator tried to draw the circle. In the experiment, the length of the discrimination data was set as $T=5$. Figure (a) shows a trace of the pointer movements, where the pointer positions are plotted in every $0.1[\mathrm{~s}]$, and the arrow indicates the pointer movement; Fig. (b) shows time histories of the EMG signals, the muscular contraction level $\alpha(n)$, the click signal, and the velocity and direction of the pointer movement. It can be seen that the direction and the velocity of the pointer movement can be voluntarily controlled by using the EMG signals. Also, the drag movements can be observed from $5.7[\mathrm{~s}]$ to $7.7[\mathrm{~s}]$.

Next, we compared the accuracy of the pointer movement by R-LLGMN with by LLGMN [3]. R-LLGMN can be regarded as an extension of LLGMN, introducing recurrent connections into LLGMN. The authors have tried to develop the EMG-controlled pointing device using LLGMN, and reported the problem of the control accuracy [5], [6]. Figure 4 (a) (b) shows the control accuracy depending on the direction. The mean values and standard deviations of the direction error are calculated for 2.0 [sec] time period. The length of the discrimination data was set as $T=1$. The experimental results shows that the estimated direction of R-LLGMN are closer to the correct values than the one of LLGMN because the direction errors are improved remarkably. The operator can control the pointer in any direction throughout 360 degrees.

\section{CONCLUSION}

In this paper, we proposed a new pointing device using the EMG signals and developed a prototype system. The proposed pointing device incorporates a neural network with a statistical structure and applies it to process EMG signals so an arbitrary direction of the pointer movement can be represented by combining finite base directions. The experiments verified that the proposed pointing device may be quite useful as a new interface tool.

Future effort will be directed to improving the system 


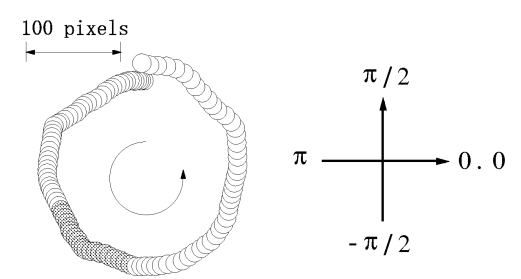

(a)

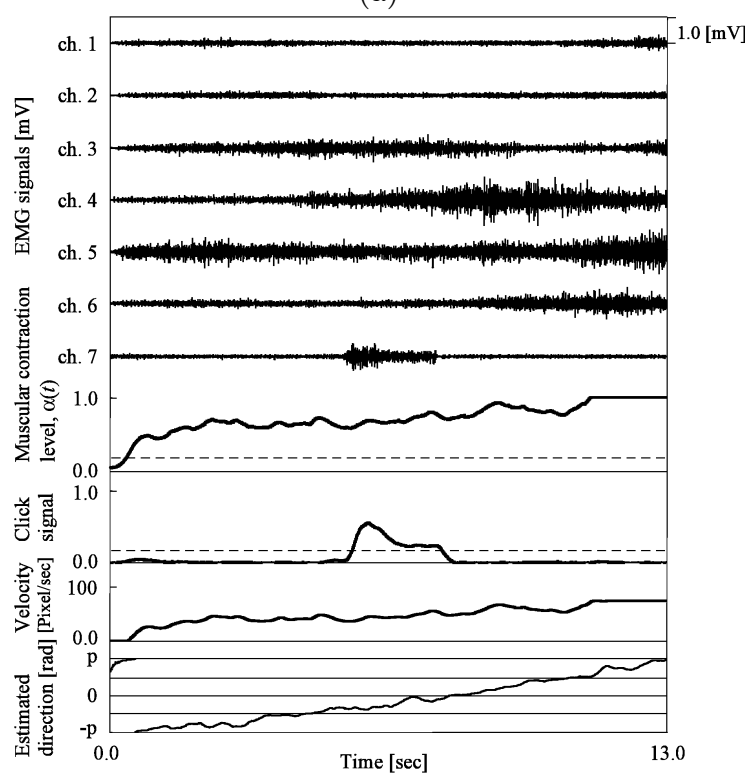

(b)

Figure 3: An example of the pointer control.

performance, such as the usage of a macro function with gesture commands.

A part of this work was supported by Industrial Technology Research Grant Program in 2001 from New Energy and Industrial Technology Development Organization (NEDO) of Japan.

\section{References}

[1] M. F. Kelly, P. A. Parker and R. N. Scott: The Application of Neural Networks to Myoelectric Signal Analysis: A Preliminary Study, IEEE Transactions on Biomedical Engineering, Vol. 37, No. 3, pp. 221-230, March, 1990.

[2] T. Tsuji, H. Ichinobe, K. Ito and M. Nagamachi: Discrimination of Forearm Motions from EMG Signals by Error Back Propagation Typed Neural Network Using Entropy, Transactions of the Society of Instrument and Control Engineers, Vol. 29, No. 10, pp. 1213-1220, 1993. (in Japanese)

[3] T. Tsuji, O. Fukuda, H. Ichinobe, M. Kaneko: "A LogLinearized Gaussian Mixture Network and Its Application to EEG Pattern Classification," IEEE Transactions on Systems, Man, and Cybernetics-Part C: Application and Reviews, Vol. 29, No. 1, pp. 60-72, February, 1999.

[4] O. Fukuda, T. Tsuji, M. Kaneko, A. Otsuka: "A HumanAssisting Manipulator Teleoperated by EMG Signals and

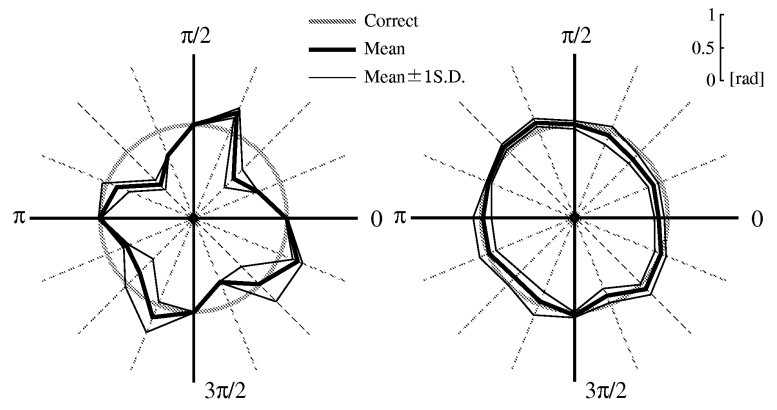

(a) LLGMN

(b) R-LLGMN

Figure 4: Accuracy of the estimated direction.

Arm Motions," IEEE Transactions on Robotics and Automation, 2003. (In press)

[5] O. Fukuda, T. Tsuji, M. Kaneko: "A Pointing Device Controlled by the EMG signals," Proceedings of SOBIM1998, pp. 281-284, 1998. (In Japanese)

[6] O. Fukuda, T. Tsuji, M. Kaneko: "An EMG-controlled Pointing Device Using a Neural Network," Proceedings of IEEE International Conference on Systems, Man and Cybernetics, Vol. 4, pp. 63-68, 1999.

[7] T. Tsuji, N. Bu, O. Fukuda, M. Kaneko: "A Recurrent Log-Linearized Gaussian Mixture Network," IEEE Transactions on Neural Networks, 2003. (In press)

[8] B.H. Juang, S.E. Levinson, and M.M. Sondhi, "Maximum Likelihood estimation for multivariate mixture observations of Markov chains," IEEE Trans. Informat. Theory, Vol. IT-32, No. 2, pp. 307-309, 1986.

[9] R. Rosenberg: "The Biofeedback Pointer: EMG Control of a Two Dimensional Pointer," Proceedings of the 2nd. International Symposium on Wearable Computers, pp. 162-163, 1998.

[10] P. M. Fitts: "The information capacity of the human motor system in controlling the amplitude of movement," Journal of Experimental Psychology, Vol. 47, pp. 381-391, 1954.

[11] A. B. Barreto, S. D. Scargle and M. Adjouadi: "A Practical EMG-based Human-computer Interface for Users with Motor Disabilities," Journal of Rehabilitation Research and Development, 37(1), pp. 53-63, 2000.

[12] J. R. Wolpaw, N. Birbaumer, W. J. Heetderks, D. J. McFarland, P. H. Packham, G. Schalk, E. Donchin, L. A. Quatrano, C. J. Robinson and T. M. Vaughan: "BrainComputer Interface Technology: A Review of the First International Meeting," IEEE Transactions on Rehabilitation Engineering, Vol. 8, No. 2, pp. 164-173, 2000.

[13] S. J. Roberts, W. D. Penny: "Real-time Brain-Computer Interfacing: a Preliminary Study Using Bayesian Learning," Medical, Biological Engineering and Computing, Vol. 38, pp. 56-61, 2000.

[14] S. G. Mason, G. E. Birch: "A Brain-Computer Switch for Asynchronous Control Applications," IEEE Transactions on Biological Engineering, Vol. 47, No. 10, pp. 1297-1307, 2000. 